\title{
De l'irrationalité de la loi de 1923 sur l'emploi des mutilés de guerre dans le service des Phares et Balises
}

Jean-Christophe Fichou

\section{(2) OpenEdition Journals}

Édition électronique

URL : http://journals.openedition.org/abpo/2737

DOI : 10.4000/abpo. 2737

ISBN : 978-2-7535-3407-0

ISSN : 2108-6443

\section{Éditeur}

Presses universitaires de Rennes

\section{Édition imprimée}

Date de publication : 27 mars 2014

Pagination : 147-165

ISBN : 978-2-7535-3405-6

ISSN : 0399-0826

\section{Référence électronique}

Jean-Christophe Fichou, «De l'irrationalité de la loi de 1923 sur l'emploi des mutilés de guerre dans le service des Phares et Balises ", Annales de Bretagne et des Pays de l'Ouest [En ligne], 121-1 | 2014, mis en ligne le 27 mars 2016, consulté le 10 décembre 2020. URL : http://journals.openedition.org/abpo/ 2737 ; DOI : https://doi.org/10.4000/abpo.2737 


\title{
De l'irrationalité de la loi de 1923 sur l'emploi des mutilés de guerre dans le service des Phares et Balises
}

\author{
Jean-Christophe FICHOU \\ Professeur CPGE au lycée de Kerichen, Brest \\ Chercheur associé au CERHIO UMR 6258 et au LÉA (Tours)
}

\section{Une Grande Guerre meurtrière}

La Première Guerre mondiale s'achève dans une odeur de sang et de mort; en France, 8410000 hommes ont été mobilisés; 1400000 d'entre eux sont morts ${ }^{1}$, auxquels s'ajoutent des centaines de milliers de blessés, de mutilés, de malades... Ces derniers, retrouvant la vie civile, doivent rechercher un emploi compatible avec leurs infirmités et l'État se doit d'aider par tous les moyens ces jeunes hommes handicapés arrivés en masse sur le marché du travail. Il faut cependant, dans un premier temps, surmonter l'absence de dispositions gouvernementales claires pour favoriser le placement de cette population amoindrie et imaginer une politique publique du handicap. Une première loi du 17 avril 1916 réserve des emplois " aux militaires et marins réformés $n^{\circ} 1$ ou retraités par suite d'infirmités résultant de blessures reçues ou de maladies contractées au cours de la guerre actuelle $^{2}$ ", mais elle est trop vague et nettement insuffisante pour répondre aux besoins. Très vite, il est décidé de réserver aux blessés de guerre une part importante des emplois, créés ou libérés, de fonctionnaires. Ainsi, " le ministre ou l'administration dont relève l'emploi doit chercher avec le ministre des anciens combattants et victimes de guerre la possibilité de la réserver en partie ou en totalité aux bénéficiaires de la présente section. Après accord, l'emploi est, le cas échéant, ajouté à ceux des tableaux sus-

1. Prost, Antoine, Les anciens combattants et la société française, 1914-1939, 1977, 3 volumes, 237, 261 et $268 \mathrm{p}$.

2. Loi du 17 avril 1916 : Droit de préférence pour l'obtention des emplois réservés dans les administrations aux anciens militaires réformés ou retraités par suite de blessures ou d'infirmités contractées durant la guerre de 1914-1918. 
visés ${ }^{3}$ ". Au ministère des Travaux publics, il est par conséquent offert des postes de gardiens de phare et c'est ainsi que des mutilés de la Grande Guerre, impotents, grabataires, se retrouvent dans les tours en mer si difficiles d'accès. Malgré toutes les interventions des ingénieurs des services maritimes pour éviter ces nominations, il faut que survienne un drame, somme toute anecdotique, au phare de la Vieille, à la pointe du Finistère, pour que l'on prenne conscience des limites de la loi sur les emplois réservés, et surtout de son application. Toute la presse s'empare de l'événement qui très rapidement s'amplifie et devient une affaire de portée nationale. L'émotion de l'opinion publique est grande et la remise en cause de la loi est jugée nécessaire pour interdire ce type d'affectation ${ }^{4}$.

\section{Des mutilés dans les administrations}

Le " droit de préférence ", pour l'obtention d'un emploi réservé aux anciens militaires réformés ou retraités par suite de blessures ou d'infirmités contractées au cours de la guerre, ne concerne que les administrations, dont le ministère des Travaux publics et le service des Phares. En ce qui concerne les emplois de gardiens de phare, un arrêté du 25 janvier 1917 complète et précise les modalités d'engagement par son article 2 :

"Avant de délivrer le certificat d'aptitude professionnelle le commandant de subdivision de région invite le candidat à se présenter à l'ingénieur des Ponts et Chaussées dans la circonscription duquel il réside afin que celui-ci puisse constater que l'intéressé est en état d'assurer le service, soit seul, soit avec l'aide de sa famille."

Très rapidement donc, les ingénieurs des arrondissements maritimes chargés d'accueillir les mutilés désireux de s'installer dans les phares prennent les précautions légales élémentaires pour éviter aux mutilés trop gravement atteints d'obtenir ces postes d'agents des phares et balises, considérés à l'époque comme de véritables sinécures. Selon le ministère de la Guerre, l'ensemble des mutilés et blessés de guerre est capable de postuler pour tous les emplois de gardiens de phares quelle que soit la nature de leurs blessures, sauf les amputés des deux membres inférieurs ou supérieurs. Pour le reste, la liste est longue..., de la perte d'un œil à l'amputation d'un membre ${ }^{5}$.

3. Article L 402 du code des pensions militaires d'invalidité et des victimes de la guerre, 2010.

4. Tous les dossiers concernant les gardiens finistériens ont été consultés en 1998 aux archives de la Délégation départementale du ministère de l'Équipement, aujourd'hui de l'Écologie, du Développement durable et de l'Énergie, alors situées à Quimper; ils ont été transférés, en partie, aux archives départementales du Finistère et sont consultables sous les cotes 22S 1 à $22 \mathrm{~S} 13$.

5. Emplois civils et militaires réservés aux militaires et marins blessés ou infirmes du fait de la guerre. Volume arrêté à la date du 18 mai 1917, Paris, Charles-Lavauzelle, 1917, p. 51. 
Et toutes ces blessures concernent un nombre d'invalides compris entre un million environ en 1924 et un maximum de 1181570 en $1933^{6}$. L'administration est parfaitement au courant des problèmes que peut soulever l'admission de candidats amoindris physiquement et dès la signature de l'armistice, les ingénieurs des Ponts et Chaussées chargés de la nomination des gardiens de phares multiplient les rapports affolés, voire alarmistes, pour demander la mise en place d'un paravent législatif efficace. Ils ne veulent, sous aucun prétexte, de mutilés dans les phares, bien que le problème ne soit pas encore trop important puisque, pour les années 1916, 1917 et 1918, on ne compte dans le Finistère qu'une dizaine de demandes de mutilés, et aucune pour les phares. En revanche, dès l'année 1919, ces dossiers s'accumulent sur les bureaux des ministères et la situation empire rapidement. Il faut placer dans une économie civile, ces PCDF comme ils se définissent, les " pauvres couillons du front ${ }^{7}$ ".

Ce n'est qu'en 1921 que les Français connaissent l'ampleur exacte de l'hécatombe après la présentation du rapport du député de Nancy, Louis Marin, qui ne le jugeait pas lui-même pleinement satisfaisant ${ }^{8}$, et selon lequel le conflit a laissé en France 3594000 blessés et environ 5000000 malades ${ }^{9}$. Une législation nouvelle s'impose pour faciliter le retour à la vie civile de ces soldats, notamment les lois du 2 mars 1916 et du 2 janvier 1918 créant l'Office national des mutilés et réformés (ONMR), établissement public rattaché au ministère du Travail et chargé de coordonner l'action entreprise par les administrations publiques en vue de l'intégration économique des blessés et mutilés de guerre ${ }^{10}$. Cet arsenal législatif est renforcé par la loi du 31 mars 1919, dite loi Lugol, sur les pensions d'invalidité en fonction du grade et du degré d'invalidité puis, par la loi du 21 juillet 1922 concernant le droit à la rééducation fonctionnelle et aux soins gratuits, et surtout la loi du 30 janvier 1923 sur les emplois réservés qui est votée dans le but d'aider les mutilés à retrouver une vie sociale ${ }^{11}$.

$\mathrm{Si}$, pour la plupart, les anciens combattants se lassèrent d'être peu écoutés et pensèrent vite à " se reposer dans la paix de la ville [...] à oublier ${ }^{12}$ ", certains ne peuvent effacer les blessures; une pension de misère, un foyer déserté, des prix multipliés par cinq, l'incompréhension de l'arrière, les rap-

6. Ministère des Pensions, Rapports de Lyons de Feuchins en 1924 et de Lebel le 20 février 1934.

7. MeYER, Jean, La vie quotidienne des soldats pendant la Grande Guerre, Paris, 1991 [1967] p. 363.

8. PRost, Antoine, "Compter les vivants et les morts : l'évaluation des pertes françaises de 1914-1918 ", Le Mouvement Social, 2008/1, p. 41-60.

9. "Les Pertes des nations belligérantes au cours de la grande guerre ", dans Les Archives de la Grande Guerre, 1921-2, t. 7, n 19, p. 37 et suivantes.

10. Loi du 2 janvier 1918 : Institution de l'Office National des Mutilés et Réformés de guerre, destiné à subventionner des écoles de rééducation.

11. Doriguzzi, Pascal, L'histoire politique du handicap. De l'infirme au travailleur handicapé. Paris, L'Harmattan, 2000, p. 97.

12. MEYER, Jean, La vie quotidienne..., op. cit., p. 365. 
pellent de toute manière à une lugubre réalité quotidienne ${ }^{13}$. Une quarantaine d'entre eux se réunissent pour la première fois en juin 1921 et créent une association, l'Union des Blessés à la Face et de la Tête, sous la direction successive du colonel Picot, de Bernard Jourdain et d'Albert Jugon. Sous le surnom des "Gueules cassées ", ils connaissent alors un véritable succès et le colonel Picot est élu député et nommé sous-secrétaire d'État. L'association pèse de tout son poids, par l'intermédiaire de ses adhérents et de son journal ${ }^{14}$, pour obliger la Chambre à voter les lois favorables aux mutilés, pour obliger le gouvernement à aider, par décrets, les anciens combattants les plus démunis par tous les moyens. Dans ce contexte, est votée la fameuse loi sur les emplois réservés, longuement préparée; le président du Conseil, Raymond Poincaré, n'appose pas sa signature au bas d'un document bâclé, mais bien d'une loi pionnière pour l'insertion des handicapés dans le monde du travail. D'ailleurs, c'est le président de la République, Alexandre Millerand, qui le paraphe en premier :

"Loi réservant des emplois aux anciens militaires pensionnés pour infirmités de guerre, ainsi qu'aux veuves et aux orphelins de guerre. Article premier - Les officiers et hommes de troupes des armées de terre et de mer, invalides de guerre, c'est-à-dire pensionnés définitifs ou temporaires, par suite de blessures reçues ou de maladies contractées ou aggravées par le fait ou à l'occasion du service au cours de la guerre 1914-1919 [...], bénéficieront pendant un délai de cinq ans [...], d'un droit de préférence pour l'obtention des emplois réservés de l'État, des établissements publics, des départements, de la ville de Paris, d'Algérie et des colonies ${ }^{15}$."

Par rapport au texte de 1916, la loi de 1923 permet à l'ensemble des victimes de guerre de bénéficier de ces emplois prioritaires : veuves et orphelins de guerre, officiers, réformés, victimes civiles ${ }^{16} \ldots$ Rien de bien nouveau cependant par rapport aux lois et décrets antérieurs puisque l'administration des Ponts et Chaussées était tenue, depuis 1848 au moins, de considérer en priorité les demandes des anciens militaires pensionnés ${ }^{17}$. Le contexte, seul, se trouve modifié dans la mesure où l'État doit favoriser le reclassement et le placement de plus de 500000 mutilés et invalides, sans

13. DELAPORTE, Sophie, "Le Retour des mutilés ", dans : Finir la guerre, actes du colloque organisé par l'association " 14-18 Meuse ", publié par le Centre d'Étude et de recherche en droit international et européen, Verdun/Nancy, Centre de recherche de l'Historial de la Grande Guerre et Université de Picardie/Presses universitaires de Nancy, 2000, 357 p.

14. Journal des Mutilés et des Réformés, 20 rue de la Chaussée d'Antin, André Linville directeur.

15. J.O. du 7 février 1923. Prost Antoine, Les anciens combattants et la société française, t. 2. Sociologie, Paris, FNSP, 1977, p. 13-18

16. Bette, Peggy, "Reclasser les victimes de la Premières Guerre mondiale. Le cas de la loi du 30 janvier 1923 sur les emplois réservés en France (1923-1939) ", dans @mins, Revue de Civilisation Contemporaine, 2006, p. 2.

17. Et cette obligation est régulièrement rappelée. Ainsi le décret du 28 juillet 1892 stipule dans son article premier : "Les emplois énumérés dans les tableaux annexés au présent règlement sont réservés aux militaires et anciens militaires remplissant les conditions fixées par l'article 81 de la loi du 15 juillet 1889. Ils ne peuvent être attribués à d'autres postulants qu'à défaut de candidats de cette catégorie. " 
compter les veuves et les orphelins ${ }^{18}$; de plus, les comités départementaux des mutilés et les associations locales de soutien veillent scrupuleusement à l'application de la loi. Bien sûr, le certificat d'aptitude reste obligatoire et deux médecins civils sont chargés d'examiner sur le plan physique les candidats en fonction de l'emploi postulé, mais le gouvernement demande beaucoup de mansuétude au corps médical car il restait essentiel pour lui que le plus grand nombre de demandes formulées puisse recevoir satisfaction :

"Aussi convient-il que, lors des épreuves subies par les candidats, [...], les intéressés soient examinés avec le plus large esprit de bienveillance [...]. Un intérêt social et patriotique de premier ordre s'attache à ce que le plus grand nombre possible de réformés [...] puissent obtenir des occupations en rapport avec leur aptitude physique et technique $[\ldots]^{19}$."

Il est vrai que les postes réservés ne présentent pas, dans l'ensemble, de difficultés particulières. On attribue plutôt des emplois calmes de portiers, de gardiens de nuit, de concierges, de garçons de salle, de surveillants d'entrepôt, de receveurs buralistes... Quelques préfectures s'inquiètent des opinions politiques des postulants, mais rapidement tout rentre dans l'ordre.

" On a demandé très récemment sans doute par erreur, des renseignements sur les opinions politiques d'un mutilé de la guerre candidat à un emploi dans l'administration des finances. Pour éviter le retour d'un incident aussi regrettable, je tiens à ce qu'il soit bien entendu que tous nos chers blessés à quelque opinion ou à quelque confession qu'ils appartiennent soient invités partout avec la même similitude ${ }^{20}$."

En revanche, dans la longue liste dressée par le ministère des Pensions, on trouve aussi des professions nettement plus physiques, et pas seulement celle des maîtres et gardiens de phares ${ }^{21}$. Les facteurs ruraux, par exemple, distribuent le courrier au cours de tournées longues de plusieurs dizaines de kilomètres. Le ministère de l'Intérieur propose des postes de gardiens de la paix; le ministère des colonies offre des postes de surveillants des établissements pénitentiaires; le ministère de l'Agriculture soumet des postes de gardes forestiers; la Marine demande des charpentiers, des tôliers, des maçons, des chaudronniers, des mécaniciens et même des artificiers!

\section{Des mutilés dans les phares en mer}

Dès juillet 1916 une liste trimestrielle des « militaires des armées de terre ou de mer, officiers et hommes de troupe, réformés $n^{\circ} 1$ ou retraités par

18. Agulhon, Maurice, Nouschi, André, Schor, Ralph, La France de 1914 à 1940, Paris, Nathan Université, 1993, p. 36.

19. Circulaire du 14 mars 1922, Paris, le ministre des Travaux publics et des Transports, Claveille.

20. Arch. dép. des Pyrénées-atlantiques, dossier gardiens, non classé. Ministère de l'Intérieur à la Préfecture des Landes, mars 1918.

21. J.O. du 7 février 1923; services divers, maîtres et gardiens de phares. Soit au total une seule et malheureuse ligne sur un ensemble de 1950 emplois répertoriés. 
suite d'infirmités résultant de blessures reçues ou de maladies contractées devant l'ennemi au cours de la guerre actuelle " parvient aux ingénieurs en chefs départementaux afin de leur signaler les emplois réclamés par les mutilés au sein de leur administration, gardiens, éclusiers, cantonniers, pontiers... En ce qui concerne le Finistère, il faut attendre la $13^{\mathrm{e}}$ liste de classement, du 7 décembre 1919, pour relever la première demande pour un emploi de gardien de phare formulée par Yves Marie Lalla, ex-soldat au $72^{\mathrm{e}} \mathrm{RI}$ amputé de deux orteils. Mais il lui est répondu qu'aucun poste n'est à pourvoir. Sur la $14^{\mathrm{e}}$ liste, du 7 mars 1920, apparaît la demande de Joseph Marie Jean, ex-second maître du $2^{\mathrm{e}}$ Dépôt des Équipages de la Flotte à Brest. La même réponse que précédemment lui est faite; il faut attendre qu'un poste se libère.

Dès lors, les ingénieurs s'inquiètent de cette recrudescence de candidats amoindris et demandent à leur ministre de prendre les dispositions nécessaires pour éviter le recrutement de gardiens trop diminués physiquement. Il ne s'agit pas d'un stratagème pour contourner les termes de la loi, comme le pratique bon nombre d'administrations ${ }^{22}$, mais bien d'une réponse à l'impossibilité physique de placer des mutilés dans les phares en mer ou dans les tours de grande hauteur. La commission de classement, sur la demande du ministre des Travaux publics, décide donc qu'un dixième seulement des vacances existant dans l'emploi de maîtres et gardiens de phares pourra être comblé par des candidats atteints d'infirmités des membres inférieurs ${ }^{23}$. De la sorte, on pense éliminer les candidats les plus estropiés. Il est clair que par tous les moyens, et dès la fin des hostilités, l'ensemble des ingénieurs des Ponts et Chaussées en poste dans tous les arrondissements maritimes dénonce la nomination des mutilés, au moins pour les emplois de gardiens.

La ligne de conduite est simple : dans un premier temps, l'ingénieur de l'arrondissement ne refuse pas les candidatures, il prévient simplement que l'attente risque de durer, une manière comme une autre de décourager les postulants et de les inciter à rechercher un autre emploi. Le second maître Jean, lassé, accepte cette solution et entreprend les démarches nécessaires pour obtenir un autre poste, mais le soldat Lalla confirme sa demande. Parfaitement conscients des risques encourus si ce gardien diminué est nommé, les ingénieurs prennent leur temps et laissent traîner l'affaire le plus longtemps possible; comme rien ne survient dans le Finistère, Lalla accepte finalement de quitter le département et obtient le poste de gardien au phare de Beauduc dans les Bouches-du-Rhône, un phare difficile perdu en Camargue. Puis les choses se précipitent. Le gardien Clet Le Gall quitte le phare en mer de la Jument décrit par l'ingénieur chargé de la construction de l'édifice comme suit :

22. BetTe, Peggy, « Les anciens combattants... », op. cit., p. 8.

23. J.O. du 29 juin 1920. 
«En raison des difficultés exceptionnelles que présente l'accostage de la Jument, et de la violence de la mer dans les parages de ce phare, il n'est pas douteux que le séjour et le service seront particulièrement pénibles et exigeront de la part du personnel beaucoup d'endurance et de dévouement. Aussi considérons-nous comme une nécessité absolue de ne nommer comme gardiens à la Jument que des hommes entraînés à des difficultés de cette nature et dont nous puissions répondre pour les avoir vus à l'œuvre, soit dans d'autres établissements, soit sur des chantiers de travaux en mer $^{24}$."

Pour le remplacer dans cet enfer de la mer d'Iroise, l'ingénieur en poste à Brest, Coÿne, propose un candidat civil, marin et ouessantin, jugé très capable, Nicolas Malgorn car ce phare " compte des conditions de séjour et de service particulièrement dures; l'emploi ne peut convenir ni à un mutilé de guerre, ni à un agent désirant changer de résidence pour raison de santé ${ }^{25}$ ". Il n'est pas question pour lui d'admettre le retour de Lalla dans cette tour où les conditions de vie sont très pénibles. Malgorn réussit à être nommé par un jeu subtil du courrier car l'ingénieur de Brest est parvenu à dépasser le délai réglementaire d'un mois pendant lequel Lalla pouvait rejoindre son affectation. Ce n'est que partie remise; dès octobre 1920 un poste se libère au phare en mer de la Vieille et Lalla argue de son classement comme mutilé pour le réclamer. L'association des Mutilés intervient d'ailleurs pour appuyer cette demande. Coÿne s'inquiète à juste raison et prévient son ingénieur en chef Lefort. Celui-ci admet facilement les arguments de son adjoint et fait part de ses craintes au directeur du service, qui est lui-même parfaitement conscient des problèmes. Lefort, ingénieur en chef, enfonce le clou et s'adresse directement au candidat :

" J'ai l'honneur d'attirer votre attention sur le poste que vous sollicitez au phare de la Vieille. Le phare, situé sur un rocher isolé en pleine mer exige des gardiens un travail très pénible qui n'est certes pas comparable à celui du phare de Beauduc où le service est beaucoup plus facile. Veuillez donc encore réfléchir ${ }^{26}$."

À son tour, l'inspecteur général et directeur des phares et balises intervient pour admettre que cet agent ne peut «faire le service de la Vieille. Il est à craindre, s'il est nommé, qu'il en résulte à bref délai des interruptions de service très préjudiciables à l'éclairage de ce phare important ${ }^{27}$ ". Pour la première fois, ce haut fonctionnaire émet même l'avis qu'il " ne devrait pas être fait de propositions en faveur de M. Lalla qu'après un examen sérieux de ce gardien ". La grande idée est lancée : d'accord pour accepter des candidats mutilés à la seule condition que leurs capacités physiques leur permettent d'assurer convenablement le service du phare où ils doivent être

24. Archives DDE Quimper, Brest, le 2 août 1911, l’ingénieur Montigny.

25. Archives DDE Quimper, Postes réservés, Brest, le 15 septembre 1920, l'ingénieur ordinaire Coÿne.

26. Archives DDE Quimper, Paris, le 15 septembre, le Directeur Ribière.

27. Archives DDE Quimper, Quimper, le 30 octobre 1920, l'ingénieur en chef Lefort. 
nommés; il convient donc de tenir compte de "la douleur des corps ${ }^{28}$ ". D'après les états nominatifs des années postérieures nous constatons que le sieur Lalla ne monta jamais à la Vieille, les ingénieurs du Finistère avaient réussi à repousser l'échéance fatale pour quelques mois encore.

Le 16 janvier 1921, le gardien Plouzennec est enlevé par une lame au phare d'Armen, et de ce fait le poste se retrouve vacant. Il convient encore une fois de nommer un candidat classé mutilé dans le phare le plus dur de tout le littoral français. Le seul qui ait formulé cette demande est François Garnier, ex-sergent au $2^{\mathrm{e}}$ RIC de Brest, pensionné à $65 \%$, mais ce dernier refuse le poste. On comprend mieux sa décision quand il nous apprend qu'il a été " blessé à Ypres le 14 décembre 1914, où une balle m'a fracassé le coude gauche [...], les nerfs coupés, ce qui occasionne une presque paralysie de tous les doigts de la main. En cette occasion, je solliciterai d'être affecté dans un poste à terre ${ }^{29}$ ". Il est remplacé par un homonyme, Émile Garnier, ex-soldat au $6^{\mathrm{e}}$ Régiment du Génie, nommé par l'arrêté ministériel du 4 mars 1921. Le 21 avril le nouveau gardien est sur la vedette de ravitaillement mais, malgré tous les efforts de l'équipage, il lui est physiquement impossible d'accéder au rocher. Le malheureux a pratiquement perdu l'usage de son bras et il faut le ligoter au cartahut pour essayer de le monter au phare. Peine perdue, il s'avère qu'une telle tentative relève plus de l'assassinat que de l'assistance aux gardiens et le canot de relève repart à Sein avec cet agent. Dans l'impossibilité de monter au phare, il sollicite une autre affectation en expliquant clairement la nature de ses blessures ${ }^{30}$.

De retour dans son département d'origine, il avertit le sénateur de Poitiers, pour lui faire part de ses déboires. Ce dernier, Guillaume Poulle, une des personnalités les plus marquantes du Sénat ${ }^{31}$, intervient auprès de l'ingénieur en chef du Finistère :

" J'ai l'honneur d'appeler particulièrement votre bienveillante attention sur le gardien Garnier Émile, nommé au phare d'Armen alors que la plus vulgaire humanité aurait dû le faire affecter à un poste en rapport avec sa situation physique. J'avoue ne pas bien comprendre comment il se fait que depuis avril dernier ce poste n'a pas pu être trouvé pour lui [...]. Je voudrais bien ne pas être obligé de porter cette question à la Tribune du Sénat ${ }^{32}$. "

Mais la menace à peine voilée n'a aucun effet puisque déjà, le 31 mai, a été nommé, toujours au phare d'Armen, le candidat classé François Hulin qui ne prend même pas possession de son poste. Le 3 septembre 1921 est nommé le candidat classé Guerrier qui lui aussi refuse ce poste. Enfin, le premier mars 1922 est nommé le candidat civil Daniel Ropart " à défaut de candidat militaire ". L'arrêté de nomination est signé le 10 mars 1922,

28. Delaporte, Sophie, " Le Corps et la parole des mutilés de la grande guerre ", dans Guerres mondiales et conflits contemporains 1/2002 ( $\left.{ }^{\circ} 205\right)$, p. 5-14.

29. Arch. dép. du Finistère, 22S 5, Brest, le 3 mars 1921.

30. Arch. dép. du Finistère, 22S 5, Brest, avril 1921.

31. Jolly, Jean, Dictionnaire des Parlementaires français, Paris, PUF, 1960-1977.

32. Arch. dép. du Finistère, 22S 5, Poitiers, le $1^{\text {er }}$ août 1921, Guillaume Poulle. 
le lendemain de la clôture de la $21^{\mathrm{e}}$ liste de classement! On note toutefois que le personnel du phare se réduit pendant plus d'un an à seulement deux gardiens titulaires avec tous les problèmes que cela pouvait supposer. Il ne faut pas croire pour autant que les candidats civils acceptent facilement l'existence dans cet enfer perché sur sa roche; pendant la même période, entre 1922 et 1924, on note les démissions successives des gardiens Jean Rohou, Noël Marie Fouquet, Le Pape et Miniou.

L'année suivante, un poste est de nouveau libéré et la commission de classement réclame la nomination d'un candidat militaire mutilé, pourtant en parfaite connaissance de cause. Jean-Marie Moalic, ancien caporal au $48^{\mathrm{e}} \mathrm{RI}$, est nommé en février 1923, mais les séquelles de ses multiples fractures à la jambe lui interdisent de monter au phare et comme Garnier il revient à terre. Il explique son geste à l'ingénieur des TPE de Brest en ces termes :

«En réponse à voter lettre du 14 février 1923, j’ai l'honneur de vous faire savoir que je suis très satisfait de ma nomination au phare d'Armen et je viens remercier M. le ministre des Travaux Publics, ainsi que M. l'ingénieur des TPE pour m'avoir nommé à un poste tant sollicité. Mais avec mon plus grand regret, je me trouve dans l'obligation de vous vous faire connaître que je suis dans l'impossibilité de prendre du service au phare d'Armen, vu la gravité de ma mutilation, fracture au tibia gauche avec raideur très serrée du genou. Mes meilleures volontés ne me permettent pas de prendre position au phare d'Armen avec une jambe raide ${ }^{33}$."

Jean-Pierre Elduayen le remplace alors, nommé par arrêté ministériel en avril, mais quand il apprend son affectation il la refuse aussitôt. Après autant de déboires, on pouvait penser que la commission avait enfin compris la situation et qu'elle accepterait les conclusions des ingénieurs locaux quant aux capacités réelles des candidats aux postes de gardiens. "Malgré toute la sollicitude dont l'Administration doit entourer les mutilés de guerre, l'intérêt supérieur du service passe avant les considérations personnelles de quiconque, si légitimes soient-elles, et il n'y a pas de raisons d'accorder aux seuls candidats militaires un privilège inacceptable ${ }^{34}$. " Pour contourner la difficulté, et la loi, les ingénieurs décident de conserver les dossiers des candidats civils sous le coude et de les présenter entre deux listes de classement, en cas de défaillance de candidats militaires.

La loi du 30 janvier 1923 ne change rien aux manières d'agir; la seule modification concerne le certificat d'aptitude professionnelle qui est délivré par une commission départementale dans laquelle siège dorénavant un conducteur des Ponts et Chaussées, Crouton, à Brest, lequel peut intervenir pour écarter des candidats jugés trop impotents. Mais sa voix n'est pas la

33. Arch. dép. du Finistère, 22S 10, Ploudaniel, le 21 février 1923.

34. Archives DDE Quimper, Brest, le 5 avril 1923, l'ingénieur Coÿne. Le lendemain, son ingénieur en chef confirme ses dires et déplore à son tour " les difficultés considérables auxquelles donnent lieu les désignations des candidats militaires et les très graves répercussions qu'elles entraînent dans le service des phares en mer qui fonctionne avec trop d'auxiliaires et trop de postes vacants ". 
seule et celles des défenseurs des mutilés est souvent plus forte, sans se soucier de la réalité de l'emploi proposé. De toute manière, les seuls postes qui se libèrent concernent uniquement des phares en mer, les plus difficiles, car par le jeu des mutations internes les postes à terre sont accordés en priorité aux agents ayant déjà effectué de nombreuses années en mer. Dans ces conditions, les mutilés ne peuvent accéder qu'à ces phares invivables, les " enfers ", lesquels demandent évidemment des qualités physiques intactes. A priori, tous les candidats classés sont rejetés par le service des Phares et seule l'insistance des autres ministères, et surtout de leurs associations de soutien, peut contraindre les ingénieurs à les accepter.

Pour se protéger légalement le directeur du service des Phares et Balises, Charles Babin, propose de soumettre au conseil d'État un projet de décret afin d'améliorer les garanties que donnent les candidats militaires. Il s'agit de tester les compétences des candidats pendant un an de stage probatoire. La mesure est acceptée par le décret du 16 mai 1924 et étendue d'ailleurs à l'ensemble des postulants, civils ou militaires. Mais les administrations gouvernementales ne peuvent toujours pas refuser les mutilés jugés aptes, d'autant plus que la loi du 26 avril 1924 étend l'emploi obligatoire des mutilés de guerre dans " toutes les exploitations industrielles et commerciales, [...] occupant régulièrement plus de dix salariés, [...] au prorata de leur personnel total ${ }^{35}$ ". Il serait mal venu pour le service des Phares de rechigner alors que l'effort national est dorénavant partagé par toutes les entreprises, publiques ou privées. De plus, il faut replacer les demandes des mutilés dans un contexte plus large; une grande souscription nationale a été ouverte à la fin de l'année 1925 par Jugon pour obtenir les fonds nécessaires à l'acquisition d'un domaine et à la construction d'un centre réservé aux "Gueules Cassées ". Dans ces conditions, il devient impossible de refuser systématiquement ces candidatures et dénoncer le regard porté sur eux plus empreint de commisération que de réalisme, mais l'absence de dispositions adaptées va se révéler cruellement à l'occasion d'un drame exploité par toute la presse locale puis nationale.

\section{Le drame de la Vieille}

Rien n'y fait : les candidats classés ne renoncent toujours pas à solliciter un poste dans les Phares et Balises, persuadés certainement de la relative quiétude de l'emploi. Charles Mondolini, né à Porto-Vecchio, ex-soldat du $17^{\mathrm{e}} \mathrm{RI}$, croix de guerre, pensionné à $40 \%$ et inscrit sur la $33^{\mathrm{e}}$ liste de classement, est nommé par arrêté ministériel le 7 mai 1925 au phare en mer de la Vieille. Averti des difficultés, il accepte pourtant cette affectation et gagne le Finistère. Le 2 juin, par très beau temps et mer calme, il rejoint son poste dans le Raz de Sein. Le patron pêcheur Pierre Coquet qui assure le

35. Loi du 26 avril 1924 assurant l'emploi obligatoire des mutilés de guerre, article 2. Pour la première fois, obligation est faite aux entreprises privées d'employer une catégorie de travailleurs. 
ravitaillement et les relèves au phare de la Vieille se rend immédiatement compte de la situation et informe le conducteur en poste à Audierne :

"Maintenant je vous dis de franche vérité, vous n'aurez pas besoin de vous étonner que la Vieille ne sera pas ravitaillée comme d'habitude quand ce sera le tour de cet homme [Mondolini] de descendre à terre ou bien de l'envoyer au phare. Jamais je n'ai vu un homme pareil. J'ai été obligé de l'amarrer comme un bidon de peur qu'il n'arrive du mal. Il tremblait avec la peur de la mer ${ }^{36}$."

Quand on apprend la nature de l'infirmité on ne s'étonne plus de l'appréhension du nouvel agent : " diminution de la capacité fonctionnelle du membre supérieur droit, suite de blessure par EO (éclats d'obus), parésie des doigts, pointe de bronchite et diminution du murmure vésiculaire. $\mathrm{Au}$ sommet gauche, séton pulmonaire par balle et manque de respiration du tiers au moindre effort ". En toute connaissance de cause, les commissions, parisienne puis brestoise, envoient au casse-pipe, dans un des phares les plus durs de France, un mutilé de guerre qui ne dispose pas, loin s'en faut, de l'intégralité de ses moyens physiques. De son côté, l'administration des Ponts, par la voix du conducteur Crouton, a vainement tenté d'interdire l'accès du phare à ce candidat. Si Mondolini est monté à la Vieille, saucissonné comme un vieux baluchon, la faute en incombe entièrement aux députés populistes et aux membres des associations de soutien aux mutilés qui poussent à cette nomination. Le drame devient inévitable car les rapports de plus en plus alarmants concernant cet agent ne parviennent toujours pas à convaincre la commission de classement de revenir sur sa décision et surtout de trouver un autre emploi à ce gardien traumatisé.

Le nouvel agent prend pourtant ses fonctions comme si de rien n'était et, dès le premier jour, il est visible que cet homme, qui ignore tout des dangers de la mer d'Iroise, n'est pas à même de remplir normalement les fonctions qui lui sont confiées.

" À son arrivée au phare, malgré qu'il fit belle mer, ce qui est extrêmement rare à ce phare, il fallut le ligoter avant que le treuil ne le soulevât de la chaloupe du ravitailleur pour le remettre au phare. C'est donc à se demander comment il pourra s'y rendre les jours de ravitaillement où la mer sera moins complaisante [...]. Certes Mondolini semble un brave garçon animé de bon vouloir mais il est inapte à seconder sérieusement, par suite de sa blessure au bras qui lui a enlevé une grande partie de sa force et sa blessure au poumon qui le menace d'une maladie de cœur aux moindres efforts. Nous demandons qu'il soit relevé, le plus tôt possible, et ceci dans son intérêt autant que dans l'intérêt du service. Ce serait criminel [...] de laisser cet homme plus longtemps occuper une situation pareille ${ }^{37}$."

Cette note est ensuite adressée au service central en rendant compte " qu'il nous est impossible de prendre plus longtemps la responsabilité du

36. Archives DDE Quimper, Postes réservés, Lescoff, le 7 juin 1925, Pierre Coquet.

37. Archives DDE Quimper, Postes réservés, Brest le 20 juin 1925, l'ingénieur des TPE Fernand Crouton. 
maintien de Mondolini au phare de la Vieille et que nous sollicitons son déplacement de toute urgence ${ }^{38}$ ». Pendant ce temps, Charles Mondolini comprend les difficultés du poste auquel il renonce. Il s'adresse au ministère des Pensions pour demander un emploi " comme facteur rural, facteur de ville ou gardien de phare à terre dans le Midi : Marseille, Toulon, Nice $^{39}$...".

Pour autant, aucune décision n'intervient et les ingénieurs locaux ne comprennent pas. Pour eux, il s'agit d'une erreur manifeste d'affectation qu'il convient de corriger au plus vite. La loi du 30 janvier 1923 stipule clairement que les mutilations doivent quand même autoriser de gravir les barreaux d'une échelle verticale en portant un fardeau et de lever un objet pondéreux au-dessus de la tête. L'article 2, dernier alinéa, prévoit d'ailleurs ce cas de figure puisqu'il énonce qu'à " titre exceptionnel, les invalides qui en raison de leur infirmité avaient été reconnus inaptes à l'emploi réservé qu'ils occupaient pouvaient en demander un autre, compatible avec leur infirmité ". Dans ces conditions pourquoi tant tarder? Mondolini doit être immédiatement mis en disponibilité avec traitement en attendant une nouvelle affectation. Le 13 août, le gardien corse descend pour la première fois de son phare afin de subir une visite médicale à Quimper, qui confirme toutes les craintes énoncées, mais il remonte quand même à son poste, dans des conditions toujours aussi dramatiques, car il ne veut et ne peut le quitter pour de basses questions matérielles. Il a déjà emprunté de l'argent pour rejoindre le Finistère et il ne sera payé que si l'arrêté ministériel reconnaît son indisponibilité. Comme le ministre des pensions n'a pris aucune décision, il reprend son service.

Le 26 novembre, il descend à terre pour la seconde fois depuis six mois, pour une autre visite médicale demandée par le ministère des pensions pour vérifier sans doute que Mondolini n'est pas un imposteur. Le certificat établi par le médecin assermenté du Finistère est bien évidemment toujours aussi sombre. Mais aucune nouvelle de la commission des emplois réservés et Mondolini remonte. Les inquiétudes se muent en malaise puis en anxiété :

" Je ne doute pas que mon Administration ait fait le nécessaire, mais n'étant toujours pas convoqué devant la commission je commence par m'inquiéter car voici l'hiver et les mauvais jours et je ne sais pas si je pourrais être capable de tenir mon poste dans un phare isolé en pleine mer, où nous sommes mal nourris et dans des conditions de vie néfastes aux invalides de guerre $^{40}$."

38. Archives DDE Quimper, Postes réservés, Brest le 22 juin 1925, l'ingénieur Coÿne et Quimper le 23 juin, l'ingénieur en chef Genet.

39. Archives DDE Quimper, Postes réservés, Phare de la Vieille, le 22 juin 1925, Charles Mondolini.

40. Archives DDE Quimper, Postes réservés, Phare de la Vieille, le 2 décembre 1925, Charles Mondolini. 
Pour simplifier la situation, en effet, le gardien classé Georges Ferraci, né aussi à Porto-Vecchio, a rejoint son compagnon d'infortune au phare de la Vieille depuis le 21 novembre. Ce dernier, inscrit sur la $35^{\mathrm{e}}$ liste de classement est un ancien soldat de la $15^{\mathrm{e}}$ section d'infirmiers militaires; il bénéficie d'une pension pour invalidité à $30 \%$. Au mois de décembre 1925 on se retrouve donc au phare avec un personnel uniquement corse, deux mutilés sans grande connaissance du monde marin et encore moins breton.

Les 2, 7, 11, 13, 21 et 22 décembre, des notes alarmantes présentées par le patron pêcheur chargé du ravitaillement, par le conducteur et par l'ingénieur ordinaire réclament la descente immédiate des deux gardiens. Le débarquement devient si dangereux que pour se soustraire à la relève tant appréhendée, Mondolini préfère passer ses congés périodiques, 10 jours par mois, sur le rocher où il vit " une existence déprimante ${ }^{41}$ ». D'ailleurs le canot de ravitaillement ne peut plus passer car une tempête terrible se lève, interdit la sortie en mer du baliseur et coupe définitivement le phare du monde des vivants. Ils ne seront relevés que le 28 février, après 88 jours au phare pour Mondolini et 45 jours pour Ferraci. Durant toute cette période le feu connaît de multiples interruptions et à partir du 16 février le pavillon de secours est hissé, mais aucune liaison n'est possible avec le phare. Le baliseur Léon Bourdelles reste à poste auprès du phare pour tenter par tous les moyens d'intervenir. Clet Coquet et son fils Pierre, les ravitailleurs sur la barque Les trois amis, se tiennent prêts aussi à assurer la relève. Le 11, un premier accostage se déroule par mer agitée, mais l'embarcation est submergée par trois paquets de mer avant d'atteindre la base du rocher, si bien qu'elle renonce à toute approche. Le 13, une nouvelle tentative s'achève par un échec tout aussi cuisant.

« Jusqu'au 26 la mer se maintint très grosse; le 26 de nouveau alerté par le pavillon hissé en berne au haut de la tour, alors que nous nous trouvions avec le baliseur dans le nord du département, nous nous rendions au pied du phare avec M. l'ingénieur des Ponts et Chaussées Coÿne qui avait tenu à venir, lui aussi tenter l'accostage. Avant d'aller au phare, nous passâmes à Sein y prendre le chef-gardien d'Armen (Nicolas Malgorn) alors à terre [...]. Notre intention était de le descendre à la Vieille pour aider le gardien Kerninon $^{42}$ à la remise en état du phare. Mais la mer était encore trop agitée pour pouvoir accoster ${ }^{43}$."

Le 28, enfin, les marins de la barque Les Trois Amis exécutent sans dommages le débarquement des gardiens Kerninon et Coquet. Une fois les deux remplaçants montés, les gardiens corses, à bout de forces, sont descendus, " pas encore sans nouveaux efforts et dangers".

41. Archives DDE Quimper, Postes réservés, Brest, le 28 décembre 1925, l'ingénieur Coÿne.

42. Jean-Noël Kerninon, troisième gardien au phare de la Vieille, nommé par arrêté ministériel à titre provisoire à Armen le $1^{\text {er }}$ octobre 1924.

43. Arch. Nat., $\mathrm{F}^{14}$ 20980, Brest, le 9 mars 1926, l'ingénieur Crouton. 
L'affaire aurait pu en rester là mais la presse s'empare de ce fait divers le 27 février, la veille du jour de la relève réussie. Alors que l'on s'inquiète du sort des deux gardiens corses, un autre drame vient secouer l'opinion. Dix jours auparavant, dans la nuit du 19 au 20 février, la goélette de Paimpol, La Surprise, sombre corps et bien dans le Raz de Sein à quelques milles du phare de la Vieille. Les neuf hommes d'équipage et le mousse périssent au cours de ce naufrage. Bien sûr, les journaux à sensation font leurs choux gras de cet accident. La Dépêche de Brest relate l'événement dans son édition du 20 février et les jours suivants apporte des précisions quant aux corps retrouvés et aux divers débris rapportés à la côte. Les journalistes prennent un malin plaisir à romancer l'événement, dépeignant la scène avec un réalisme morbide, décrivant minutieusement les cadavres rejetés nus sur le sable de la baie des Trépassés, allant jusqu'à évoquer un cœur arraché de la poitrine d'un matelot. Le dimanche 27 février, l'accusation est lancée sur fond de rumeur : "Plusieurs fois déjà le phare s'est éteint et le bruit court, à la Pointe du Raz, que pendant la nuit où se perdit la Surprise, la trompe de brume de la Vieille ne résonnait pas ${ }^{44}$."

Sur place, les marins interrogés confirment et racontent que depuis la Noël le phare de la Vieille s'est maintes fois éteint et que la sirène de brume ne fonctionne pas avec régularité. Il n'en faut pas davantage pour que les journalistes locaux fassent le lien entre ces défaillances humaines et le naufrage de La Surprise. Au cours de leur enquête, ils apprennent la sombre tragédie qui se joue au phare de la Vieille et s'emparent du fait divers tragique pour rédiger un long article, très documenté, mais truffé d'erreurs. Ainsi, l'administration centrale des Phares est nommément accusée de ne pas avoir tenu compte des certificats médicaux présentés par les deux gardiens corses. Ce qui est faux, comme nous l'avons expliqué. Plus grave, les auteurs de l'article soutiennent que la corne de brume ne fonctionnait pas le soir du drame. L'édition du 28 février est beaucoup moins polémique et rétablit une certaine vérité :

«[...] au service des phares, certes, on n'a pas manqué de tenir compte de l'incompatibilité existant entre l'état d'un mutilé et le gardiennage d'un phare; mais on n'en put tenir compte que pour le signaler aux administrations supérieures. Et l'on se heurtait ainsi à un droit absolu : les emplois de gardiens de phares étaient strictement réservés aux mutilés ${ }^{45}$ "

Alertée, la presse parisienne s'empare dès lors de l'affaire en fustigeant le ministre des Travaux publics qui a osé autoriser la montée des deux mutilés au phare ${ }^{46}$.

"Trois mois d'angoisse - Deux mutilés dans l'enfer ${ }^{47}$. "

44. La Dépêche de Brest, le 26 février 1926.

45. La Dépêche de Brest, le 28 février 1926.

46. La Dépêche de Brest est le correspondant local du quotidien parisien, L'Intransigeant, qui reprend le premier dans la capitale l'histoire tragique des mutilés corses en noircissant encore un plus les faits présentés par les journalistes brestois.

47. Journal des Mutilés \& Réformés, le 6 mars 1926. 
"Les emplois réservés, les leçons du phare de la Vieille. L’Administration a, on le voit, tout ce qu'il faut pour se défendre. Et bien elle n'a fait en ce qui concerne les emplois de gardiens de phare aucune réserve, vous entendez bien, aucune réserve. Et elle a embarqué comme cela sur la simple référence de leur bonne volonté des mutilés la veille cultivateurs et la veille comme le lendemain malheureusement physiquement diminués ${ }^{48}$."

Le premier mars, on apprend que deux députés corses, messieurs Cailucoli et de Moro-Giafferi ${ }^{49}$ viennent de déposer une demande d'interpellation au parlement sur le drame qu'avaient vécu leurs deux compatriotes. L'affaire par la suite se tasse et l'on oublie peu à peu les deux compères corses de la Vieille. Rentrés à Brest le 2 mars, nos deux gardiens subissent une nouvelle visite médicale qui nous les dépeint en très mauvais état : " Le régime déprimant qu'ils eurent à soutenir durant un long laps de temps fait que leur santé, déjà affaiblie par leurs blessures de guerre, est devenue précaire ainsi que l'attestent les certificats médicaux ${ }^{50}$. " Ferracci est examiné au centre anti-tuberculeux de Brest où le diagnostic est édifiant :

« Diagnostic : insuffisance respiratoire avec séquelles de congestion pleuro-pulmonaire gauche; présence d'un corps étranger et ralentissement notable de l'appareil cardio-musculaire. Impropre au service des phares. Le médecin directeur Bodros ${ }^{51}$."

Par arrêté ministériel en date du 3 avril 1926, la mise en disponibilité avec traitement complet est prononcée pour raisons de santé avec traitement entier. Deux mois supplémentaires leur sont accordés par la décision du 8 mai et ensuite, comme tous les fonctionnaires, ils ne sont plus rémunérés qu'à demi-traitement. Charles Mandolini obtient enfin un poste de facteur rural à Bretteville-sur-Laize en mai 1927; quant au sort de Charles Ferraci, les archives restent muettes, et la famille contactée n'a pu nous renseigner. Une place de garde dans les Eaux et Forêts est demandée, mais elle n'est pas obtenue, semble-t-il.

\section{Modifier la loi}

Pour autant, le problème général n'est toujours pas légalement réglé dans la mesure où la loi de janvier 1923 n'est pas abrogée; et dorénavant tous les départements littoraux sont concernés. Le candidat militaire, François Clair, nommé à Cordouan en avril 1926, puis Trompeloup, deux phares de Gironde, démissionne en mars 1928; il s'agit d'un "réformé de guerre dont l'état de santé n'a pas permis de continuer à tenir l'emploi ${ }^{52}$ ".

48. Journal des Mutilés \& Réformés, 20 mars 1926.

49. Vincent de Moro-Giafferi (1878-1956), grand avocat d'assises et homme politique français d'origine corse. Il est resté célèbre, entre autres, pour avoir défendu Henri Landru, condamné à mort et exécuté en février 1922.

50. Archives DDE Quimper, Brest, le 5 mars 1926, l'ingénieur Crouton.

51. Arch. dép. du Finistère, 22S 5, Brest, le 4 mars 1926, dispensaire de Keroriou.

52. Arch. dép. de Gironde. Dossiers du port autonome de Bordeaux, n 299, Bordeaux, le 17 mars 1928. 
Pierre Rossi, ancien soldat du $81^{\mathrm{e}}$ régiment d'infanterie, candidat militaire classé, est nommé le $1^{\mathrm{er}}$ novembre 1928 au phare des Barges, en face des Sables-d'Olonne, mais il ne rejoint pas son poste; la nomination est rapportée. Dans le département de la Manche, Jean Ruellen, né à Guisseny, est nommé gardien en juin 1926 au phare en mer de La Hague. Ce candidat militaire, décoré de la médaille militaire, de la croix de guerre avec palme, présente une blessure au genou droit paraissant avoir sectionné le ligament latéral externe. Finalement, il refuse son poste et s'en explique auprès de l'ingénieur de Cherbourg :

"Monsieur le sous ingénieur, n'ayant pu aller jusqu'à votre bureau avant de partir, je vous écris ces quelques mots pour vous faire savoir dans quelles circonstances j'ai quitté le phare de la Hague. Le poste me plaisait très bien, mais pour moi, je n'aurais jamais fait qu'un malheureux là dedans vu que ma plus grande peine c'est de monter les escaliers et justement il y en a beaucoup. Cherbourg, le 3 juillet $1926^{53}$."

La commission des Phares se doit de réagir rapidement et au cours de la séance du 19 mars 1926, elle émet le vœu " qu'une modification du régime en vigueur pour le recrutement des gardiens de phares soit poursuivie d'urgence par les départements ministériels compétents ", après avoir constaté l'inaptitude des agents nommés au titre militaire. Les conseils régionaux maritimes prennent les mêmes dispositions; dans les Bouchesdu-Rhône, lors de la séance du 11 octobre 1926, le problème est soulevé car de nombreux abandons ont été notifiés par des mutilés nommés dans les trois phares de Camargue, Beauduc, Faraman et La Gacholle qui, bien que situés à terre, sont parmi les plus durs de France. Après une courte discussion, le conseil général,

" considérant que l'affectation des mutilés de guerre dans les services de gardiens de phares est préjudiciable à la bonne marche de ce service important, émet le vœu que ces fonctions ne soient confiées à l'avenir qu'à des agents offrant toutes garanties physiques et professionnelles.

Demande aux parlementaires de vouloir faire solutionner cette ques$\operatorname{tion}^{54}$."

Le $1^{\text {er }}$ septembre 1927, le ministère des Travaux publics présente un nouveau texte beaucoup plus restrictif quant au recrutement qui doit écarter définitivement les mutilés de guerre. Décret, suivi d'un arrêté fixant les conditions dans lesquelles devront être subies les épreuves d'aptitude professionnelle des candidats aux postes de gardien de phare en mer. Mais, dans le même temps, est votée le 21 juillet 1928 une loi imposant la présence d'un invalide de guerre au sein des commissions médicales et professionnelles de classement, renforçant ainsi leurs droits. Il semble bien d'ailleurs que ce soient ces derniers textes qui aient le plus d'effet

53. Arch. dép. de la Manche, 1S Granville 8, Dossiers individuels - Gardiens de phares (1873-1929).

54. Conseil général du département des Bouches-du-Rhône, Rapports et délibérations 1926, Marseille, Imprimerie nouvelle, 1927, p. 1268. 
puisque nous apprenons que Philippe Paollacci, né à Casevecchio en Corse et ancien soldat du 55 $\mathrm{RI}$, est nommé en octobre 1927 à la Vieille où il remplace un autre mutilé, Dolimpas. Le 20 février 1928, l'ingénieur Crouton est en tournée au phare :

"Le gardien Paolacci était au phare et nous lui avons trouvé une si mauvaise mine que nous lui avons enjoint de descendre à terre au prochain ravitaillement afin que nous le fassions examiner du point de vue de son état de santé [...] D'excellent caractère, mais d'une paresse de couleuvre, paresse peut-être due à son état de santé, Paolacci est incapable du moindre effort ni capable de tirer sur un câble, ni capable de mettre le moteur de brume en route. Le pauvre malheureux a insisté près de nous pour avoir son changement dans le Midi si possible. Nous en informons le service, car c'est tout ce que nous pouvons faire ${ }^{55}$."

Finalement, il obtient un congé de 12 mois et il est nommé, enfin, garçon de bureau à la préfecture de Rodez le 5 décembre $1929^{56}$. Nous savons aussi que deux autres Corses pensionnés sont nommés dans le Finistère, en 1927 et en 1928, alors que les ingénieurs départementaux, indignés, dénoncent ce qui leur apparaît comme un scandale fondé sur une effroyable confusion :

« Depuis 1925, les phares d'Armen et de la Vieille ont connu une succession de mutilés de guerre corses : Secondi, Mandolini, Ferracci, Paollacci... qui tous à peine rendus en service dans leur phare en mer ne pensent qu'à une chose, c'est d'en partir le plus tôt possible. Loin de leur pays où ils laissent leur famille, privés des soins de leur épouse, vivant sous un climat humide et dans des tours de phare encore plus humides, s'alimentant mal, leur moral décline très vite et leurs blessures et leur santé s'altèrent et se ravivent. C'est encore le cas de Del Guidice. Ce sera encore le cas de celui qui le remplacera si c'est un Corse $^{57}$."

Finalement, le service des Phares n'obtient gain de cause qu'en novembre 1931. Par arrêté ministériel, on fixe une fois pour toutes le programme des aptitudes physiques nécessaires pour postuler un emploi de gardien de phare. Les candidats doivent " savoir nager (cent mètres) et conduire une embarcation (aviron et godille) ${ }^{58}$ ". Les conditions d'embauche sont encore renforcées en mars 1932, après la signature de l'arrêté ministériel fixant les aptitudes physiques auxquelles doivent satisfaire les candidats à l'emploi de gardien de phare ${ }^{59}$. Ainsi, à Rennes, les services médicaux des Armées sont chargés de vérifier les qualités physiques de deux candidats mutilés pour un poste de gardiens de phare :

55. Arch. dép. du Finistère, 22S 11, Brest, le 23 février 1928.

56. Arch. dép. du Finistère, 22S 11, Brest, le 23 février 1928.

57. Arch. dép. du Finistère, 22S 4, Brest, le 4 février 1929, l'ingénieur TPE Crouton.

58. Arrêté ministériel du 30 novembre 1931, Paris, le ministre des Travaux publics, Maurice Deligne.

59. Arrêté ministériel du 18 mars 1932, Paris, le ministre des Travaux publics, Charles Guernier. 
" M. le général, Commandant le $2^{\mathrm{e}}$ groupe de subdivision à Rennes, a demandé à notre service de faire subir à MM. Van Assche et Leroux, candidats à des emplois réservés, les épreuves techniques relatives à un emploi de gardien de phare. [l'examen] a révélé que ces candidats sont manifestement inaptes à la fonction de gardiens de phares, tant au point de vue des travaux manuels que des connaissances pratiques en mécanique et électricité ${ }^{60}$. "

À l'inverse, en septembre 1935, Amédé Le Pape, jeune candidat, se présente à son tour au centre de Saint-Nazaire pour passer les épreuves de sélection :

" Il a gravi avec facilité les barreaux d'une échelle verticale de $4 \mathrm{~m}$ en portant un poids de $15 \mathrm{~kg}$ de cordage, puis il a levé au-dessus de sa tête un objet de $2 \mathrm{~kg}$. Il est monté au sommet des haubans du mât du Paul Leferme, soit $12 \mathrm{~m}$, avec aisance. Il est monté très rapidement dans la lanterne du phare du Vieux-Môle de Saint-Nazaire. Il ne présentait aucun signe d'emphysème. Il sait nager. Il sait conduire une embarcation à la godille aussi bien qu'à l'aviron ${ }^{61}$."

Il est clair dorénavant que plus aucun mutilé vraiment diminué ne peut obtenir ce type de poste dans les phares en mer, mais il fallut attendre dix ans et connaître un drame de mer au retentissement national pour parvenir à une législation adaptée.

"Comment peut-on vivre dans certains phares qui jalonnent l'Atlantique finistérien? On n'a peut être oublié ce qui se passa, il y a sept ans, au phare de la Vieille qui est l'autre nom de Gorlébella. Il se dresse en plein Raz de Sein, en plein fleuve Océan, en pleins remous. Les gardiens étaient deux Corses, mutilés de guerre. La France reconnaissante leur avait octroyé, comme situation de tout repos cette fonction... Une loi bien intentionnée attribuait à des blessés de guerre un certain nombre de postes, comme s'il s'agissait de squares ou de bureaux ${ }^{62} \ldots$ "

Il a fallu le caractère inédit de la présence massive de ces jeunes hommes handicapés sur le marché du travail pour que la perception du handicap soit profondément modifiée. Cet exemple des mutilés de la Vieille, somme toute anecdotique, est portant très évocateur, d'une part, de la volonté affirmée des anciens combattants pour retrouver une place dans la société d'après-guerre. Et, d'autre part, cette histoire ubuesque et plus encore sa conclusion, fruit du hasard d'une disposition administrative mal pensée dans l'application de la loi, confirment l'idée d'une évolution rapide de la perception du handicap, de "l'infirme au travailleur handicapé ${ }^{63}$ ". Il n'est plus question seulement d'assistance, de légitimation des droits dus

60. Arch. dép. d'Ille-et-Vilaine, 1S 102, Saint-Malo, le 6 janvier 1933, Rapport du subdivisionnaire.

61. Archives des Phares et balises de Saint-Nazaire; résultats des épreuves passées le 17 septembre 1935.

62. Dupouy, Auguste, Face au couchant, Brest, la côte et les îles, Quimper, Caligramme, 1984 [1934], p. 46.

63. DoriguZZI, P., L'Histoire politique du handicap..., op. cit. 
au sacrifice de ces soldats, mais bien de la prise en compte d'un état, de la reconnaissance du handicap et de l'acceptation des différences.

\section{RÉSUMÉ}

Après le Première Guerre mondiale, les autorités françaises cherchent à placer les mutilés de guerre. Tous les ministères doivent leur offrir des postes de fonctionnaires adaptés à leur situation et jugés de tout repos, tout du moins compatibles avec leur handicap. Le ministère des Travaux publics n'est pas en reste et plus particulièrement le service des Phares et balises qui est lui aussi mis à contribution et sommé d'accueillir en son sein ces soldats blessés, comme toutes les autres administrations françaises. Très vite cependant le directeur de ce service tente de se soustraire à cette obligation car il considère, à juste titre d'ailleurs, inconcevable d'attribuer des postes de gardiens à des agents amoindris physiquement. Il faut un drame national pour que l'opinion et le Parlement prennent conscience de l'absurdité de la situation et n'impose plus la présence de ces anciens soldats blessés dans des tours en mer, isolées et souvent battues par les tempêtes.

\section{ABSTRACT}

After the First World War, the French authorities sought to find employment for mutilated war veterans. All ministries were forced to offer civil-service positions that were adapted to their situation and often considered to be soft jobs or, in any event, compatible with their disabilities. The ministry of public labour and, in particular, the Department of Lighthouses and Beacons was also required to welcome wounded soldiers and provide jobs, just as were all other sections of the French administration. Rapidly, however, the director of this department tried to escape from this obligation as he considered, quite rightly, that it was inconceivable to appoint physically diminished employees as wardens. It was only when a national tragedy ensued that public opinion and parliament realised the absurdity of the situation and released the department from the obligation of employing wounded war veterans in isolated and often windswept lighthouses. 